MATHEMATICS OF COMPUTATION

Volume 75 , Number 253 , Pages $307-326$

S 0025-5718(05)01774-6

Article electronically published on October 4, 2005

\title{
ON COMPUTING RATIONAL GAUSS-CHEBYSHEV QUADRATURE FORMULAS
}

\author{
JORIS VAN DEUN, ADHEMAR BULTHEEL, AND PABLO GONZÁLEZ VERA
}

\begin{abstract}
We provide an algorithm to compute the nodes and weights for Gauss-Chebyshev quadrature formulas integrating exactly in spaces of rational functions with arbitrary real poles outside $[-1,1]$. Contrary to existing rational quadrature formulas, the computational effort is very low, even for extremely high degrees, and under certain conditions on the poles it can be shown that the complexity is of order $O(n)$. This method is based on the derivation of explicit expressions for Chebyshev orthogonal rational functions, which are (thus far) the only examples of explicitly known orthogonal rational functions on $[-1,1]$ with arbitrary real poles outside this interval.
\end{abstract}

\section{INTRODUCTION}

Over the last few years, several generalizations of the classical Gauss quadrature formulas have been studied, e.g., formulas which integrate exactly in spaces of Laurent polynomials 1, 5, 6, 13 or more general rational functions 2, 3, 11, 12, 16, 17. However, most of these formulas suffer from a serious drawback, i.e., the computational effort required to construct the formulas is so large that it is usually not compensated for by their superior accuracy for specific integrands. The same is true in the case of (polynomial) Gauss formulas for arbitrary measures, whose computation was discussed in a series of articles by Gautschi [7, 8, 9, 10]. To construct these formulas, the general procedure is the same for polynomials, Laurent polynomials or rational functions: one first has to compute the recurrence coefficients, and then obtain the nodes and weights from these coefficients. If the coefficients are not explicitly known, they can be computed evaluating the inner products in the expressions for the coefficients, and this is where many of the problems arise. Basically, to approximate a given integral, you want to use a quadrature formula whose construction involves the evaluation of many very similar integrals (inner products); you would need a very good reason to do things this way. Some notable exceptions to all this are the (few) formulas which can be computed from explicitly known modified moments [8], some of the formulas based

Received by the editor August 5, 2004.

2000 Mathematics Subject Classification. Primary 42C05, 65D32.

Key words and phrases. quadrature formulas, orthogonal rational functions.

The work of the first author was partially supported by the Fund for Scientic Research (FWO), projects "CORFU: Constructive study of orthogonal functions", grant \#G.0184.02 and, "RAM: Rational modelling: optimal conditioning and stable algorithms", grant \#G.0423.05, and by the Belgian Programme on Interuniversity Attraction Poles, initiated by the Belgian Federal Science Policy Office. The scientific responsibility rests with the author.

(C)2005 American Mathematical Society Reverts to public domain 28 years from publication 
on Laurent polynomials from [13] and of course the explicitly known formulas such as the classical Chebyshev rule.

The main purpose of this paper is to present a class of gaussian quadrature formulas on $[-1,1]$, based on orthogonal rational functions, which can be computed fast and efficiently (even for very high degrees). They are rational generalizations of the Gauss-Chebyshev formula, and are exact in a maximal space of rational functions with arbitrary real poles outside $[-1,1]$, which are fixed in advance. Unlike the polynomial case, the weights and nodes are not explicitly known, but are easy to compute. In fact, the computations are simplified considerably by "translating" them from the interval to the complex unit circle, as was done in [14. To arrive at these formulas, we first have to generalize the Chebyshev polynomials to the case of orthogonal rational functions and thereby provide the only explicitly known examples of orthogonal rational functions on $[-1,1]$ with arbitrary real poles outside this interval.

In the next section we present the basic theory of orthogonal rational functions (ORF). The main ingredients here are the three-term recurrence relation and the gaussian quadrature formulas. In Section 3 we specialize this theory to the case of Chebyshev weight functions, using a theorem from Szegö's book 15. We give explicit representations for the ORF and their recurrence coefficients. The computation of the quadrature formula is discussed in Section 4, and in the Examples section we look at the computation and accuracy of these formulas and compare them to some alternatives.

\section{Preliminaries}

The complex plane is denoted by $\mathbb{C}$, the Riemann sphere by $\overline{\mathbb{C}}=\mathbb{C} \cup\{\infty\}$, the real line by $\mathbb{R}$ and the extended real line by $\overline{\mathbb{R}}=\mathbb{R} \cup\{\infty\}$. For the unit circle and its interior we introduce the following notation:

$$
\mathbb{T}=\{z:|z|=1\}, \quad \mathbb{D}=\{z:|z|<1\} .
$$

We will also use $I=[-1,1], \overline{\mathbb{R}}^{I}=\overline{\mathbb{R}} \backslash I$ and $\overline{\mathbb{C}}^{I}=\overline{\mathbb{C}} \backslash I$. Given a positive bounded Borel measure $\mu$ on $I$ whose support $\operatorname{supp}(\mu) \subset I$ is an infinite set, the inner product is defined as

$$
\langle f, g\rangle=\int_{-1}^{1} f(x) \overline{g(x)} d \mu(x) .
$$

Next we will introduce the spaces of rational functions with real poles. Let a sequence $A=\left\{\alpha_{1}, \alpha_{2}, \ldots\right\} \subset \overline{\mathbb{R}}^{I}$ be given. Define factors

$$
Z_{n}(x)=\frac{x}{1-x / \alpha_{n}}, \quad n=1,2, \ldots,
$$

and basis functions

$$
b_{0}=1, \quad b_{n}(x)=b_{n-1}(x) Z_{n}(x), \quad n=1,2, \ldots
$$

Then the space of rational functions with poles in $A$ is defined as

$$
\mathcal{L}_{n}=\operatorname{span}\left\{b_{0}, \ldots, b_{n}\right\} .
$$

Let $\mathcal{P}_{n}$ denote the space of polynomials of degree at most $n$ and define

$$
\pi_{n}(x)=\prod_{k=1}^{n}\left(1-x / \alpha_{k}\right) .
$$


Then we may write equivalently

$$
\mathcal{L}_{n}=\left\{p_{n} / \pi_{n}, p_{n} \in \mathcal{P}_{n}\right\} .
$$

Note that $\mathcal{L}_{n}=\mathcal{P}_{n}$ if all poles are at infinity. Orthonormalizing the canonical basis $\left\{b_{0}, \ldots, b_{n}\right\}$ with respect to the orthogonality measure $\mu$, we obtain orthogonal rational functions $\left\{\varphi_{0}, \ldots, \varphi_{n}\right\}$. Regarding these orthonormal functions we have the following important theorem from [2, p. 261], completely analogous to the one for orthogonal polynomials. The regularity conditions mentioned in 2 are automatically satisfied because of the assumption on the location of the poles.

Theorem 2.1. Put by convention $\alpha_{-1}=\alpha_{0}=\infty$. Then for $n=1,2, \ldots$ there exist constants $E_{n}, F_{n}$ such that the orthonormal rational functions $\varphi_{n}$ satisfy the following three-term recurrence relation:

$$
\varphi_{n}(x)=\left(E_{n} Z_{n}(x)+F_{n} \frac{Z_{n}(x)}{Z_{n-1}(x)}\right) \varphi_{n-1}(x)-\frac{E_{n}}{E_{n-1}} \frac{Z_{n}(x)}{Z_{n-2}(x)} \varphi_{n-2}(x) .
$$

The initial conditions are $\varphi_{-1}(x) \equiv 0, \varphi_{0}(x) \equiv 1 / \sqrt{\mu(I)}$, and the coefficients $E_{n}$ are nonzero.

Note that the coefficient $E_{0}$ is never used and can be arbitrarily chosen. If we take the coefficient $E_{n}$ to be positive, then the functions $\varphi_{n}$ will be uniquely determined. In this case we have the following lemma which can be deduced from [2].

Lemma 2.2. The orthonormal functions $\varphi_{n}$ normalized with $E_{n}>0$ have real coefficients with respect to the basis $\left\{b_{k}\right\}$.

It follows in particular that $\varphi_{n}(x)$ is real for real $x$, and for any inner product $\left\langle f, \varphi_{n}\right\rangle$ we may omit the complex conjugate bar in (2.1).

The reproducing kernel $k_{n}(x, y)$ for the space $\mathcal{L}_{n}$ is defined by

$$
k_{n}(x, y)=\sum_{k=0}^{n} \varphi_{k}(x) \overline{\varphi_{k}(y)},
$$

and the orthogonal rational functions $\varphi_{n}$ satisfy a Christoffel-Darboux relation, which we will only need in its confluent form as given in the next theorem from 2 , Chap. 11].

Theorem 2.3. The following relation holds between reproducing kernel and orthonormal basis functions of $\mathcal{L}_{n}$ :

$$
f_{n}^{\prime}(x) f_{n-1}(x)-f_{n}(x) f_{n-1}^{\prime}(x)=E_{n} k_{n-1}(x, x),
$$

where

$$
f_{k}(x)=\left(1-\frac{x}{\alpha_{k}}\right) \varphi_{k}(x)
$$

and the prime means derivative.

Next we give the general theorem about rational gaussian quadrature formulas. It can be found in [2, chapter 11.6], and in more detail in [17.

Theorem 2.4. 1. For each $n=1,2, \ldots$, the function $\varphi_{n}(z)$ has exactly $n$ distinct zeros on $(-1,1)$. 
2. Let $\left\{x_{n k}\right\}_{k=1}^{n}$ be the zeros of the $n$-th orthogonal rational function $\varphi_{n}$ and let $\left\{\lambda_{n k}\right\}_{k=1}^{n}$ be defined by

$$
\lambda_{n k}=k_{n-1}\left(x_{n k}, x_{n k}\right)^{-1} .
$$

Then the quadrature formula for

$$
I_{\mu}(f)=\int_{-1}^{1} f(x) d \mu(x)
$$

given by

$$
I_{n}(f)=\sum_{k=1}^{n} \lambda_{n k} f\left(x_{n k}\right)
$$

is exact in $\mathcal{L}_{n} \cdot \mathcal{L}_{n-1}=\left\{g h: g \in \mathcal{L}_{n}, h \in \mathcal{L}_{n-1}\right\}$, i.e., $I_{n}(f)=I_{\mu}(f)$ for any $f$ in $\mathcal{L}_{n} \cdot \mathcal{L}_{n-1}$.

It follows from 4 that the nodes can be found as the eigenvalues of a generalized eigenvalue problem and that the weights are defined by the first components of the corresponding eigenvectors. The matrices involved contain the recurrence coefficients and the poles.

We conclude this section with a theorem about the asymptotic zero distribution of the functions $\varphi_{n}(x)$. It can be found in [16. We rephrase it according to our discussion.

Theorem 2.5. Let $\mu$ be absolutely continuous, satisfying the Erdös-Turán condition $\mu^{\prime}>0$ a.e. on $I$, and suppose that the asymptotic distribution of the poles $A$ is given by a measure $\nu$ on $\overline{\mathbb{R}} \backslash I$, i.e., for every continuous function $f$ on $\overline{\mathbb{R}} \backslash I$ which vanishes at infinity, we have

$$
\lim _{n \rightarrow \infty} \frac{1}{n} \sum_{j=1}^{n} f\left(\alpha_{j}\right)=\int_{\overline{\mathbb{R}} \backslash I} f(x) d \nu(x) .
$$

If $\nu=p \delta_{\infty}+(1-p) \nu_{0}$, where $\delta_{\infty}$ is a unit measure with all its mass concentrated at infinity and $0 \leq p \leq 1$ (note that $p$ measures the ratio of the number of poles at infinity to the total number of poles), and

$$
\int_{\overline{\mathbb{R}} \backslash I} \log |t| d \nu_{0}(t)<\infty,
$$

then the asymptotic distribution of the zeros of $\varphi_{n}$ is given by an absolutely continuous measure $\lambda$ with weight function

$$
\lambda^{\prime}(x)=\frac{1}{\pi} \frac{1}{\sqrt{1-x^{2}}} \int_{\overline{\mathbb{R}} \backslash I} \frac{\sqrt{1-1 / t^{2}}}{1-x / t} d \nu(t) .
$$

\section{Chebyshev ORF}

Contrary to the polynomial case with its classical examples such as the Legendre or Chebyshev polynomials, no explicit examples of orthogonal rational functions on $I$ are known for arbitrary poles. In fact, this lack of "classical" cases is one of the most poignant problems in the computational theory of ORF. In this section we derive explicit formulas for a rational generalization of the Chebyshev polynomials, which is an important first step in this direction. In what follows, by a Chebyshev weight function we mean a Jacobi weight function of the form $w(x)=$ $(1-x)^{\alpha}(1+x)^{\beta}$ with $\alpha$ and $\beta$ belonging to $\{ \pm 1 / 2\}$. Furthermore, note that the 
case $\alpha=-1 / 2$ and $\beta=1 / 2$ reduces to the case $\alpha=1 / 2$ and $\beta=-1 / 2$ replacing $x$ by $-x$. The key to most results is a theorem which can be found in Szegö's book [15. p. 31]. Let $\rho(x)$ be a polynomial of strict degree $l$ and positive in $[-1,1]$. Then this theorem gives explicit representations for polynomials $p_{n}(x)$ orthogonal with respect to the weight functions

$$
w(x)=\left\{\begin{array}{l}
\left(1-x^{2}\right)^{-1 / 2}\{\rho(x)\}^{-1}, \\
\left(1-x^{2}\right)^{1 / 2}\{\rho(x)\}^{-1}, \\
\left(\frac{1-x}{1+x}\right)^{1 / 2}\{\rho(x)\}^{-1} .
\end{array}\right.
$$

These orthogonal polynomials $p_{n}(x)$ are called Bernstein-Szegö polynomials and can be given explicitly provided that $l<2 n$ in the first case, $l<2(n+1)$ in the second case and $l<2 n+1$ in the third. We give the theorem without proof.

Theorem 3.1. Let $\rho(x)$ be a polynomial of strict degree $l$ and positive in $[-1,1]$. Let $\rho(\cos \theta)=\left|h\left(e^{\mathbf{i} \theta}\right)\right|^{2}$ be the normalized representation of $\rho(\cos \theta)$ such that $h(z)$ is a polynomial of degree $l$, with $h(z) \neq 0$ in $\mathbb{D}$ and $h(0)>0$. Then we have the following formulas for the orthonormal polynomials $p_{n}(x)$, orthogonal in the sense that $\int_{-1}^{1} p_{k}(x) p_{l}(x) w(x) d x=\delta_{k l}$ :

$$
\begin{aligned}
p_{n}(\cos \theta) & =(2 / \pi)^{1 / 2} \Re\left\{e^{\mathbf{i} n \theta} \overline{h\left(e^{\mathbf{i} \theta}\right)}\right\}, & \\
w(x) & =\left(1-x^{2}\right)^{-1 / 2}\{\rho(x)\}^{-1}, & l<2 n ; \\
p_{n}(\cos \theta) & =(2 / \pi)^{1 / 2}(\sin \theta)^{-1} \Im\left\{e^{\mathbf{i}(n+1) \theta} \overline{h\left(e^{\mathbf{i} \theta}\right)}\right\}, & \\
w(x) & =\left(1-x^{2}\right)^{1 / 2}\{\rho(x)\}^{-1}, & l<2(n+1) ; \\
p_{n}(\cos \theta) & =\pi^{-1 / 2}(\sin (\theta / 2))^{-1} \Im\left\{e^{\mathbf{i}(n+1 / 2) \theta} \overline{h\left(e^{\mathbf{i} \theta}\right)}\right\}, & \\
w(x) & =\left(\frac{1-x}{1+x}\right)^{1 / 2}\{\rho(x)\}^{-1}, & l<2 n+1 .
\end{aligned}
$$

The connection with orthogonal rational functions is as follows. If we denote as usual the orthonormal rational functions with respect to $\mu$ by $\varphi_{n}(x)$, then writing $\varphi_{n}(x)=p_{n}(x) / \pi_{n}(x)$, it follows that $p_{n}(x)$ is a polynomial orthogonal (not orthonormal) to the varying measure $d \mu(x) /\left(\pi_{n}(x) \pi_{n-1}(x)\right)$. As pointed out by Van Assche in [16], when $d \mu(x)=w(x) d x$ with

$$
w(x)=\left\{\begin{array}{l}
\left(1-x^{2}\right)^{-1 / 2} \\
\left(1-x^{2}\right)^{1 / 2} \\
\left(\frac{1-x}{1+x}\right)^{1 / 2}
\end{array}\right.
$$

then we can use the previous theorem to compute the polynomials $p_{n}$ (and thus also the rational functions $\varphi_{n}$ ).

Before we can proceed with our discussion, some lemmas are needed which simplify the computations. In the rest of this chapter we will use the following definition for Blaschke factors $\zeta_{k}(z)$ and the corresponding Blaschke product $B_{n}(z)$. Let there be given a sequence of real numbers $B=\left\{\beta_{1}, \beta_{2}, \ldots\right\} \subset I$. Then define

$$
\begin{aligned}
\zeta_{k}(z) & =\frac{z-\beta_{k}}{1-\beta_{k} z}, \quad k=1,2, \ldots \\
B_{n}(z) & =B_{n-1}(z) \zeta_{n}(z), \quad B_{0}(z)=1, \quad n=1,2, \ldots
\end{aligned}
$$

The first lemma computes an integral that we will encounter later on. 
Lemma 3.2. With the previous definition of the Blaschke product $B_{n}(z)$ we have

$$
\int_{0}^{2 \pi} \frac{\left[z^{k} B_{n}(z) B_{n-1}(z) \pm 1\right]^{2}}{\left(z-\beta_{n}\right)^{2} B_{n-1}^{2}(z) z^{k-1}} d \theta= \pm \frac{4 \pi}{1-\beta_{n}^{2}} \quad\left(z=e^{\mathbf{i} \theta}\right)
$$

for every $k=1,2, \ldots$

Proof. First expand the square in the numerator to get

$$
\begin{aligned}
\int_{0}^{2 \pi} \frac{\left[z^{k} B_{n}(z) B_{n-1}(z) \pm 1\right]^{2}}{\left(z-\beta_{n}\right)^{2} B_{n-1}^{2}(z) z^{k-1}} d \theta=\int_{0}^{2 \pi} \frac{z^{k+1} B_{n}^{2}(z)}{\left(z-\beta_{n}\right)^{2}} d \theta \\
\pm 2 \int_{0}^{2 \pi} \frac{z}{\left(z-\beta_{n}\right)\left(1-\beta_{n} z\right)} d \theta+\int_{0}^{2 \pi} \frac{1}{\left(z-\beta_{n}\right)^{2} B_{n-1}^{2}(z) z^{k-1}} d \theta .
\end{aligned}
$$

Note that the last integral in this expression is the complex conjugate of the first one, so we may write

$$
\begin{aligned}
& \int_{0}^{2 \pi} \frac{\left[z^{k} B_{n}(z) B_{n-1}(z) \pm 1\right]^{2}}{\left(z-\beta_{n}\right)^{2} B_{n-1}^{2}(z) z^{k-1}} d \theta \\
&=2 \Re\left\{\frac{1}{\mathbf{i}} \oint_{\mathbb{T}} \frac{z^{k} B_{n}^{2}(z)}{\left(z-\beta_{n}\right)^{2}} d z\right\} \pm \frac{2}{\mathbf{i}} \oint_{\mathbb{T}} \frac{1}{\left(z-\beta_{n}\right)\left(1-\beta_{n} z\right)} d z .
\end{aligned}
$$

The first integrand is analytic in $\mathbb{D} \cup \mathbb{T}$, and the second one has a simple pole in $z=\beta_{n}$. The result now follows from the residue theorem.

As indicated by this lemma, Blaschke products have a peculiar behaviour when $z$ is on the unit circle. For example, in the proof we used the fact that $\overline{B_{n}(z)}=$ $1 / B_{n}(z)$ when $z \in \mathbb{T}$. The next lemma gives an interesting expression for the derivative of a Blaschke product on the unit circle.

Lemma 3.3. With the definition of the Poisson kernel

$$
P(z, t)=\frac{1-|t|^{2}}{|z-t|^{2}}, \quad z \in \mathbb{T}, \quad t \notin \mathbb{T},
$$

the derivative $B_{n}^{\prime}(z)$ can be written

$$
B_{n}^{\prime}(z)=\frac{1}{z} B_{n}(z) \sum_{k=1}^{n} P\left(z, \beta_{k}\right)
$$

whenever $z \in \mathbb{T}$.

Proof. Note that with $z \in \mathbb{T}$ we have

$$
\zeta_{n}^{\prime}(z)=\frac{1}{z} \zeta_{n}(z) P\left(z, \beta_{n}\right)
$$

The result is now immediate using the definition of the Blaschke product.

We denote the Joukowski transform $x=\frac{1}{2}\left(z+z^{-1}\right)$ by $x=J(z)$, mapping the open unit disc $\mathbb{D}$ to the cut Riemann sphere $\overline{\mathbb{C}}^{I}$ and the unit circle $\mathbb{T}$ to the interval $I$. The inverse mapping is denoted by $z=J^{-1}(x)$ and is chosen so that $z \in \mathbb{D}$ if $x \in \overline{\mathbb{C}}^{I}$. Given a weight function $w(x)$ on $I$, define the weight $\stackrel{\circ}{w}(\theta)$ on $[0,2 \pi)$ as follows:

$$
\stackrel{\circ}{w}(\theta)=w(\cos \theta)|\sin \theta| .
$$


This implies that for an arbitrary function $f$ we have

$$
\int_{-1}^{1} f(x) w(x) d x=\frac{1}{2} \int_{0}^{2 \pi} f(\cos \theta) \stackrel{\leftrightarrow}{w}(\theta) d \theta .
$$

Now we are ready to give explicit expressions for orthogonal rational functions with respect to the weights in (3.1). Assume as before that we have arbitrary real poles $\alpha_{k}$ outside $I$. We use the notation $\varphi_{n}^{(i)}, i=1,2,3$, to denote the orthogonal rational functions with respect to the $i$-th weight in (3.1), so, e.g., $\varphi_{n}^{(1)}$ denotes the rational generalization of the classical Chebyshev polynomials. However, we will use the name "Chebyshev orthogonal rational functions" for all three cases. They are given in the next theorem.

Theorem 3.4. The orthonormal functions $\varphi_{n}^{(i)}$ are given by

$$
\begin{aligned}
& \varphi_{n}^{(1)}(x)=\frac{1}{\sqrt{2 \pi}} \sqrt{1-\beta_{n}^{2}}\left(\frac{z B_{n-1}(z)}{1-\beta_{n} z}+\frac{1}{\left(z-\beta_{n}\right) B_{n-1}(z)}\right), \\
& \varphi_{n}^{(2)}(x)=\sqrt{\frac{2}{\pi}} \sqrt{1-\beta_{n}^{2}} \frac{z}{z^{2}-1}\left(\frac{z^{2} B_{n-1}(z)}{1-\beta_{n} z}-\frac{1}{z B_{n-1}(z)\left(z-\beta_{n}\right)}\right), \\
& \varphi_{n}^{(3)}(x)=\frac{1}{\sqrt{\pi}} \frac{1}{z-1} \sqrt{1-\beta_{n}^{2}}\left(\frac{z^{2} B_{n-1}(z)}{1-\beta_{n} z}-\frac{1}{\left(z-\beta_{n}\right) B_{n-1}(z)}\right),
\end{aligned}
$$

where $x=J(z) \in \overline{\mathbb{C}}$ and $\alpha_{k}=J\left(\beta_{k}\right)$.

Proof. From the definition of $\pi_{n}(x)$ at the beginning of Section 2 we see that

$$
\pi_{n}(\cos \theta)=\left|\prod_{k=1}^{n} \frac{1-\beta_{k} z}{\sqrt{1+\beta_{k}^{2}}}\right|^{2}, \quad z=e^{\mathbf{i} \theta}
$$

The polynomial between the absolute value signs obviously satisfies the conditions for the normalized representation of $\pi_{n}$ as mentioned in Theorem 3.1. Using that theorem and the remark following it, we then find that

$$
p_{n}^{(1)}(x)=c_{n}^{(1)} \sqrt{\frac{2}{\pi}} \Re\left\{z^{-n+1} \prod_{k=1}^{n-1} \frac{\left(z-\beta_{k}\right)^{2}}{1+\beta_{k}^{2}} \frac{\left(z-\beta_{n}\right)}{\sqrt{1+\beta_{n}^{2}}}\right\},
$$

where $\varphi_{n}^{(1)}(x)=p_{n}^{(1)}(x) / \pi_{n}(x)$ and $c_{n}^{(1)}$ is a normalization constant such that

$$
\int_{-1}^{1} \frac{\left(\varphi_{n}^{(1)}(x)\right)^{2}}{\sqrt{1-x^{2}}} d x=1
$$

Dividing by $\pi_{n}(x)$ as given in (3.3) and using the definition of the Blaschke products yields

$$
\begin{aligned}
\varphi_{n}^{(1)}(x) & =c_{n}^{(1)} \sqrt{\frac{2}{\pi}} \Re\left\{\frac{z B_{n-1}(z)}{1-\beta_{n} z} \sqrt{1+\beta_{n}^{2}}\right\} \quad\left(x=\cos \theta, z=e^{\mathrm{i} \theta}\right) \\
& =c_{n}^{(1)} \frac{1}{\sqrt{2 \pi}} \sqrt{1+\beta_{n}^{2}}\left(\frac{z B_{n-1}(z)}{1-\beta_{n} z}+\frac{1}{\left(z-\beta_{n}\right) B_{n-1}(z)}\right) .
\end{aligned}
$$

The second equation holds for $x$ anywhere in the complex plane and not just on I. To find the constant $c_{n}^{(1)}$, use equations (3.4) and (3.2) and Lemma 3.2. This completes the proof for $\varphi_{n}^{(1)}(x)$. The other two cases are proved in a completely analogous way. 
Using the explicit expressions we can derive many other formulas, e.g., for the nodes and weights in the quadrature formulas. This will be done in the next section. First we look at the recurrence coefficients.

Theorem 3.5. For $n>1$ the recurrence coefficients as for the $\varphi_{n}^{(i)}(x)$ are the same as for the three different cases and are given by

$$
\begin{aligned}
& E_{n}=2 \frac{\sqrt{\left(1-\beta_{n-1}^{2}\right)\left(1-\beta_{n}^{2}\right)}\left(1-\beta_{n-1} \beta_{n}\right)}{\left(1+\beta_{n-1}^{2}\right)\left(1+\beta_{n}^{2}\right)} \\
& F_{n}=-\sqrt{\frac{1-\beta_{n}^{2}}{1-\beta_{n-1}^{2}} \frac{\left(1-\beta_{n-1}^{2}\right)\left(\beta_{n}+\beta_{n-2}\right)+2 \beta_{n-1}\left(1-\beta_{n} \beta_{n-2}\right)}{\left(1+\beta_{n}^{2}\right)\left(1-\beta_{n-1} \beta_{n-2}\right)}}
\end{aligned}
$$

where $\beta_{k}=J^{-1}\left(\alpha_{k}\right)$. For $n=1$ we have

$$
\begin{array}{lll}
E_{1}^{(1)}=\sqrt{2} c, & E_{1}^{(2)}=2 c, & E_{1}^{(3)}=2 c \\
F_{1}^{(1)}=-\sqrt{2} \beta_{1} c, & F_{1}^{(2)}=-\beta_{1} c, & F_{1}^{(3)}=\left(1-\beta_{1}\right) c
\end{array}
$$

where

$$
c=\frac{\sqrt{1-\beta_{1}^{2}}}{1+\beta_{1}^{2}} .
$$

For $n=2$ the formula for $F_{2}$ holds with the convention that $\beta_{0}=0$.

Proof. The coefficients can be computed using the formulas

$$
\begin{aligned}
E_{n} & =\lim _{x \rightarrow \alpha_{n-1}} \frac{\varphi_{n}(x)}{\varphi_{n-1}(x) Z_{n}(x)} \\
F_{n} & =\lim _{x \rightarrow \alpha_{n-2}}\left(\frac{\varphi_{n}(x)}{\varphi_{n-1}(x)} \frac{Z_{n-1}(x)}{Z_{n}(x)}-E_{n} Z_{n-1}(x)\right) .
\end{aligned}
$$

These formulas are valid for $n \geq 1$ (with the usual convention that $\alpha_{-1}=\alpha_{0}=\infty$ ), but the case $n=1$ has to be treated separately because the expressions for $\varphi_{n}^{(i)}(x)$ as given by Theorem 3.4 do not include $\varphi_{0}^{(i)}$. For the case $n>1$ equations (3.5) and (3.6) can easily be verified. If $n=1$, then use the fact that $\varphi_{0}^{(i)} \equiv 1 / \sqrt{\mu_{i}(I)}$, where $\mu_{i}$ refers to the corresponding weight function. To compute $F_{1}^{(i)}$ the simpler formula

$$
F_{1}^{(i)}=\frac{\varphi_{1}^{(i)}(0)}{\varphi_{0}^{(i)}}
$$

can be used. Some computations now finish the proof.

Remark 3.6. Note that we get the recurrence coefficients for the Chebyshev polynomials if we take all poles at infinity (which corresponds to taking all $\beta_{k}$ 's equal to zero). Furthermore, the values given by (3.5) and (3.6) are exactly the asymptotic values from Theorem 7 in [18]. In this sense, Chebyshev ORF are indeed a natural generalization of Chebyshev polynomials. It is a well-known fact that the recurrence coefficients for general orthogonal polynomials on $I$ with respect to a measure $\mu$ satisfying $\mu^{\prime}>0$ a.e. behave asymptotically like the coefficients of Chebyshev polynomials.

Once the recurrence coefficients are known, we can use the generalized eigenvalue problem mentioned in the previous section to compute the nodes and weights in the gaussian quadrature formulas. However, next we derive alternative expressions to 
compute these values, which are far more efficient, especially for very large values of $n$. Several examples in Section 5 will illustrate this.

\section{QuAdrature FORMULAS}

We finally arrive at the main purpose of this paper, which is the construction of rational Gauss-Chebyshev quadrature formulas. The nodes in these formulas are the zeros of $\varphi_{n}^{(i)}(x)$. In the polynomial case, these zeros can easily be expressed in closed form. Thus, for instance, the zeros of the $n$-th Chebyshev polynomial of the first kind $(\alpha=\beta=1 / 2)$ are given by

$$
x_{n k}=\cos \left(\pi \frac{2 k-1}{2 n}\right), \quad k=1,2, \ldots, n .
$$

It is difficult (or even impossible) to give similar explicit representations for the zeros of Chebyshev ORF, but we can derive formulas which allow an efficient computation of these zeros, as shown in the next theorem.

Theorem 4.1. Let $x_{n k}^{(i)}$ denote the zeros of $\varphi_{n}^{(i)}(x)$ and put $x_{n k}^{(i)}=\cos \theta_{n k}^{(i)}$. Then they satisfy the equations

$$
\begin{aligned}
f_{n}\left(\theta_{n k}^{(1)}\right)-(n-1) \theta_{n k}^{(1)} & =\frac{\pi}{2}(2 k-1), \\
f_{n}\left(\theta_{n k}^{(2)}\right)-(n-2) \theta_{n k}^{(2)} & =\pi k, \\
f_{n}\left(\theta_{n k}^{(3)}\right)-(n-3 / 2) \theta_{n k}^{(3)} & =\pi k,
\end{aligned}
$$

for $k=1,2, \ldots, n$, where

$$
f_{n}(\theta)=2 \sum_{j=1}^{n-1} \arctan \frac{\sin \theta}{\cos \theta-\beta_{j}}+\arctan \frac{\sin \theta}{\cos \theta-\beta_{n}},
$$

and $\arctan (y / x)$ refers to the argument of the complex number $x+\mathbf{i} y$.

Proof. Again we only prove the theorem for the first case. From the expression for $\varphi_{n}^{(1)}(x)$ we get that the zeros satisfy

$$
z_{n k}^{(1)} B_{n}\left(z_{n k}^{(1)}\right) B_{n-1}\left(z_{n k}^{(1)}\right)=-1=e^{\mathbf{i} \pi(2 k-1)}, \quad k \in \mathbb{Z},
$$

where $z_{n k}^{(1)}=J^{-1}\left(x_{n k}^{(1)}\right)$. So we see that the function $z B_{n}(z) B_{n-1}(z)+1$ should have its $2 n$ zeros on $\mathbb{T}$. Furthermore, they are pairwise complex conjugates. Taking the real parts gives us the $n$ zeros of $\varphi_{n}^{(1)}(x)$. Note that $\zeta_{n}(z)$ may be written as

$$
\zeta_{n}(z)=\exp \left\{2 \mathbf{i} \arctan \frac{\sin \theta}{\cos \theta-\beta_{n}}-\mathbf{i} \theta\right\}, \quad z=e^{\mathbf{i} \theta} .
$$

Some computations complete the proof. Note that for the second case the function $z^{3} B_{n}(z) B_{n-1}(z)-1$ has zeros at -1 and 1 which cancel against the same zeros in the denominator of $\varphi_{n}^{(2)}(x)$ and which therefore have to be ignored. The same occurs in the third case for $z=1$.

In general, the equations from this theorem cannot be solved analytically. It is possible, however, to calculate the nodes in the quadrature formulas numerically using Newton's method, as described below. First we need the following simple lemma. 
Lemma 4.2. The functions $f_{n}(\theta)-(n-c) \theta$ from Theorem 4.1 with $c \in\{1,3 / 2,2\}$ are strictly increasing for $0 \leq \theta \leq \pi$. Furthermore, if all poles have equal sign, these functions are concave (positive poles) or convex (negative poles) on $(0, \pi)$. In general there can be at most one interior inflection point.

Proof. The first statement follows from the fact that

$$
\frac{d}{d \theta}\left(\arctan \frac{\sin \theta}{\cos \theta-\beta}\right)=\frac{1-\beta \cos \theta}{1-2 \beta \cos \theta+\beta^{2}} \geq \frac{1}{1+|\beta|}>\frac{1}{2}
$$

for $|\beta|<1$. This means that $f_{n}^{\prime}(\theta)-(n-c)>c-1 / 2$, which is strictly positive for all three cases. To prove the second statement, note that

$$
\frac{d^{2}}{d \theta^{2}}\left(\arctan \frac{\sin \theta}{\cos \theta-\beta}\right)=\sin \theta \frac{\beta\left(\beta^{2}-1\right)}{\left(1-2 \beta \cos \theta+\beta^{2}\right)^{2}} .
$$

This means that $f_{n}^{\prime \prime}(\theta)=\sin \theta \tilde{f}_{n}(\theta)$, where $\tilde{f}_{n}(\theta) \leq 0$ on $[0, \pi]$ for positive poles or $\tilde{f}_{n}(\theta) \geq 0$ on $[0, \pi]$ for negative poles. If there are both positive and negative poles, differentiating once more shows that $\tilde{f}_{n}(\theta)$ is a monotonic function on $(0, \pi)$, which means that it can have at most one zero there.

To illustrate this lemma, Figure 1 shows the function $f_{n}(\theta)-(n-1) \theta$ for the case where $\beta_{2 k-1}=0.9$ and $\beta_{2 k}=-0.8$ for $k=1,2, \ldots$, and $n=100$.

Newton's method for finding zeros works particularly well for monotonic functions, especially if the initial values are not too far from the exact solutions. Next we discuss two different methods for determining these initial values.

The first method is based on linear extrapolation. Let $\left\{\theta_{n k}\right\}_{k=1}^{n}$ denote the $n$ exact zeros. For the case where all poles are positive, the initial value $\theta_{n, k+1}^{0}$ for the zero $\theta_{n, k+1}$ is determined from the previous two zeros as

$$
\theta_{n, k+1}^{0}=\theta_{n k}+\left(\theta_{n k}-\theta_{n, k-1}\right) .
$$

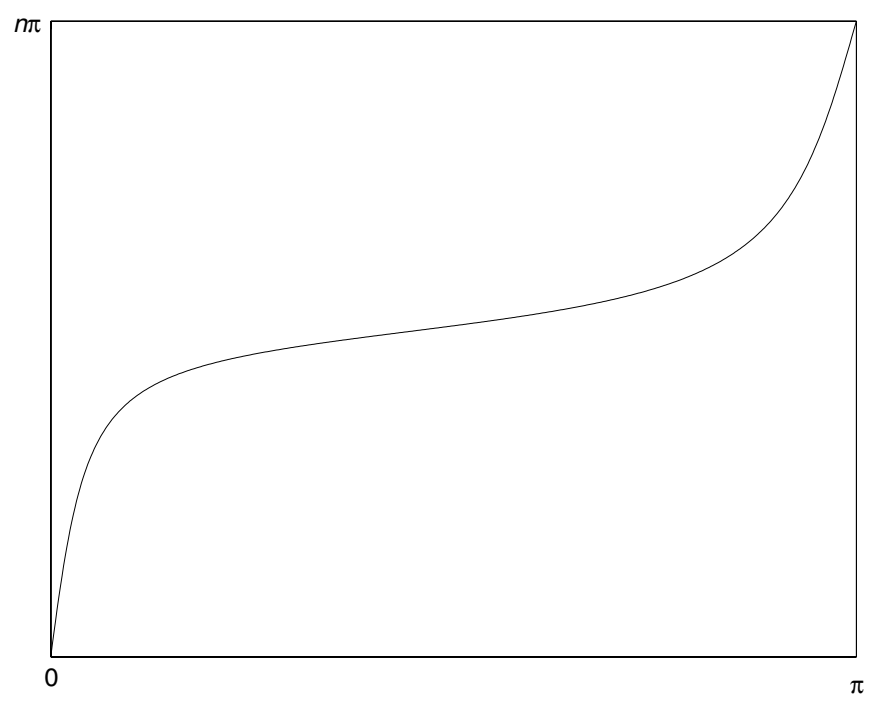

Figure 1. The function $f_{n}(\theta)-(n-1) \theta$. 
We work from left to right taking $\theta_{n 1}^{0}=\theta_{n 0}=0$. For a strictly increasing concave function, Newton's method converges monotonically (i.e., the successive iterations form an increasing sequence) if $\theta_{n, k+1}^{0}<\theta_{n, k+1}$. This condition is satisfied because of the linear extrapolation and Lemma 4.2. The monotonic convergence guarantees that the iterations cannot fall outside the interval $[0, \pi]$, so we do not have to check this, which may save some time.

If all poles are negative, the procedure is similar, but now we take

$$
\theta_{n, k-1}^{0}=\theta_{n k}+\left(\theta_{n k}-\theta_{n, k+1}\right),
$$

working from right to left with $\theta_{n n}^{0}=\theta_{n, n+1}=\pi$.

In general, when there are both positive and negative poles, either of the above procedures can be used. Of course, one could devise a strategy taking into account the number of positive and negative poles respectively, but we do not do this.

The second method for determining the initial values is based on the asymptotic zero distribution as given by Theorem 2.5. First we assume that the poles tend to a fixed limit with increasing $n$, i.e., $\lim _{n \rightarrow \infty} \alpha_{n}=\alpha$, so that the zero distribution is given by a measure $\lambda$ whose derivative is equal to

$$
\lambda^{\prime}(x)=\frac{1}{\pi} \frac{1}{\sqrt{1-x^{2}}} \frac{\sqrt{1-1 / \alpha^{2}}}{1-x / \alpha} .
$$

The zero density on the interval $[-1, x]$ equals

$$
t(x)=\int_{-1}^{x} \lambda^{\prime}(u) d u=\frac{1}{\pi} \arcsin \frac{\alpha x-1}{\alpha-x}+\frac{1}{2} .
$$

Solving for $x$ gives

$$
x=\frac{1-\alpha \cos (\pi t)}{\alpha-\cos (\pi t)}
$$

so if we take $n$ equally distributed numbers $t_{k n} \in[0,1]$ as

$$
t_{k n}=\frac{2 k-1}{2 n}, \quad k=1, \ldots, n,
$$

then we can estimate the zeros $x_{k n}$ by plugging the values $t_{k n}$ into equation (4.1). Note that for $\alpha=\infty$ this gives us exactly the zeros of the Chebyshev polynomial $T_{n}(x)$.

For the more general case of arbitrary poles, we cannot use this procedure. In practice we are given only a finite number of poles, so we do not know anything about the asymptotic behaviour of the poles. However, we can approximate the zero density $t(x)$ by the finite sum

$$
t_{n}(x)=\frac{1}{\pi n} \sum_{j=1}^{n} \arcsin \frac{\alpha_{j} x-1}{\alpha_{j}-x}+\frac{1}{2},
$$

which, in fact, converges pointwise to the exact zero density $t(x)$ if $n \rightarrow \infty$. The inverse function can no longer be found analytically, but we compute a spline approximation as follows. First, for a suitable value of $m$, we evaluate the function $t_{n}(x)$ in the zeros

$$
\xi_{k}=\cos \left(\pi \frac{2 k-1}{2 m}\right)
$$

of the Chebyshev polynomial $T_{m}(x)$. These zeros cluster near the endpoints of the interval $[-1,1]$, where $t_{n}(x)$ is steep (especially if the poles are close to the interval). 
The function $t_{n}^{-1}(t)$ behaves more like a cosine than like a polynomial — in fact, for all poles at infinity, we have $t_{n}^{-1}(t)=-\cos \pi t-$ so to obtain an approximation to $t_{n}^{-1}(t)$ we use a cubic interpolating spline $s(t)$ through the points $\left(t_{n}\left(\xi_{k}\right), \arccos \xi_{k}\right)$. The initial values for the zeros $\theta_{k n}$ are then given by

$$
\theta_{k n}^{0}=s\left(t_{k n}\right) \text {, }
$$

where $t_{k n}$ are the points from equation (4.2). More details are given in the Examples section.

The advantage of this method is that it gives us all initial values at the same time, so we can apply Newton's method to all zeros at once (at least if the implementation is done in a language which supports vector operations, such as Matlab or Fortran). Depending on the hardware and the specific implementation, this can save a considerable amount of time. Of course the estimates will be better for larger $n$ because the method is based on asymptotic behaviour.

Once the nodes have been computed, the weights can be found by evaluating the kernel function as explained in Section 2. Since we have explicit formulas for the orthogonal functions, this is a straightforward task. However, it is possible to give much simpler expressions for the weights, as shown in the following theorem.

Theorem 4.3. The weights in the gaussian quadrature formulas based on the $\varphi_{n}^{(i)}$ can be given as functions of the nodes as

$$
\begin{aligned}
& \lambda_{n k}^{(1)}=2 \pi\left(1+g_{n}\left(x_{n k}^{(1)}\right)\right)^{-1}, \\
& \lambda_{n k}^{(2)}=2 \pi\left(1-\left(x_{n k}^{(2)}\right)^{2}\right)\left(3+g_{n}\left(x_{n k}^{(2)}\right)\right)^{-1}, \\
& \lambda_{n k}^{(3)}=2 \pi\left(1-x_{n k}^{(3)}\right)\left(2+g_{n}\left(x_{n k}^{(3)}\right)\right)^{-1}
\end{aligned}
$$

for $k=1,2, \ldots, n$, where

$$
g_{n}(x)=2 \sum_{k=1}^{n-1} \frac{\sqrt{1-1 / \alpha_{k}^{2}}}{1-x / \alpha_{k}}+\frac{\sqrt{1-1 / \alpha_{n}^{2}}}{1-x / \alpha_{n}} .
$$

Proof. We give the proof for the first case. To simplify the notation, we omit the superscripts, so we write $\varphi_{n}$ instead of $\varphi_{n}^{(1)}$ and also we write $x_{k}$ instead of $x_{n k}^{(1)}$, etc. The proof is based on the confluent Christoffel-Darboux formula from Theorem 2.3. It follows from this theorem and the formula for the weights in the quadrature formula that

$$
\left(1-\frac{x_{k}}{\alpha_{n}}\right) \varphi_{n}^{\prime}\left(x_{k}\right) \varphi_{n-1}\left(x_{k}\right)\left(1-\frac{x_{k}}{\alpha_{n-1}}\right)=\frac{E_{n}}{\lambda_{k}} .
$$

First note that for any $x \in I$,

$$
\left(1-\frac{x}{\alpha_{n}}\right)\left(1-\frac{x}{\alpha_{n-1}}\right)=\frac{\left|1-\beta_{n} z\right|^{2}}{1+\beta_{n}^{2}} \frac{\left|1-\beta_{n-1} z\right|^{2}}{1+\beta_{n-1}^{2}},
$$

where $x=J(z)$. Using the formula for $\varphi_{n}$ from Theorem 3.4 and the chain rule for differentiation gives

$$
\varphi_{n}^{\prime}(x)=\frac{1}{\sqrt{2 \pi}} \sqrt{1-\beta_{n}^{2}}\left[\frac{z B_{n}(z) B_{n-1}(z)+1}{\left(z-\beta_{n}\right) B_{n-1}(z)}\right]^{\prime} \frac{2 z^{2}}{z^{2}-1},
$$


which, using Lemma 3.3 and the fact that $x_{k}$ is a zero of $\varphi_{n}$, can be written as

$$
\begin{aligned}
\varphi_{n}^{\prime}\left(x_{k}\right)= & \frac{1}{2 \pi} \sqrt{1-\beta_{n}^{2}} \frac{B_{n}\left(z_{k}\right)}{\left(z_{k}-\beta_{n}\right)} \\
& \times\left[1+2 \sum_{j=1}^{n-1} P\left(z_{k}, \beta_{j}\right)+P\left(z_{k}, \beta_{n}\right)\right] \frac{2 z_{k}^{2}}{z_{k}^{2}-1} .
\end{aligned}
$$

It follows from the fact that $z_{k} B_{n}\left(z_{k}\right) B_{n-1}\left(z_{k}\right)+1=0$ that

$$
\begin{aligned}
\frac{B_{n}\left(z_{k}\right)}{\left(z_{k}-\beta_{n}\right)} & {\left[\frac{z_{k} B_{n-2}\left(z_{k}\right)}{1-\beta_{n-1} z_{k}}+\frac{1}{\left(z_{k}-\beta_{n-1}\right) B_{n-2}\left(z_{k}\right)}\right] } \\
& =\frac{\left(z_{k}^{2}-1\right)\left(1-\beta_{n} \beta_{n-1}\right)}{z_{k}^{2}\left|1-\beta_{n} z_{k}\right|^{2}\left|1-\beta_{n-1} z_{k}\right|^{2}}
\end{aligned}
$$

which gives

$$
\varphi_{n-1}\left(x_{k}\right) \frac{B_{n}\left(z_{k}\right)}{\left(z_{k}-\beta_{n}\right)}=\frac{1}{\sqrt{2 \pi}} \sqrt{1-\beta_{n-1}^{2}} \frac{\left(z_{k}^{2}-1\right)\left(1-\beta_{n} \beta_{n-1}\right)}{z_{k}^{2}\left|1-\beta_{n} z_{k}\right|^{2}\left|1-\beta_{n-1} z_{k}\right|^{2}} .
$$

Combining this formula with equations (4.3)-(4.5) and the formula for $E_{n}$ gives, after cancelling terms,

$$
\lambda_{k}=2 \pi\left[1+2 \sum_{j=1}^{n-1} P\left(z_{k}, \beta_{j}\right)+P\left(z_{k}, \beta_{n}\right)\right]^{-1} .
$$

Transforming back to $x_{k}$ and $\alpha_{j}$ then proves the theorem for the first case. The other two cases are proved in the same way.

To conclude this section we look at the computational complexity of constructing these quadrature formulas.

Theorem 4.4. Let there be $m$ different poles among the first $n$ poles. Then it will take $O(m n)$ operations to compute the nodes and weights in the quadrature formulas of this section. In particular, if all poles are different, the complexity is of order $O\left(n^{2}\right)$, while if there is only a finite number of different poles ( $m$ does not depend on $n$ for large $n)$, then the complexity is of order $O(n)$.

Proof. If there are only $m$ different poles among the first $n$, then evaluating the functions $f_{n}, f_{n}^{\prime}$ and $g_{n}$ from Theorems 4.1 and 4.3 takes $O(m)$ operations (we have to sum over $m$ different values instead of $n$ ). Determining the initial values using the asymptotic zero distribution is also of order $O(m)$, for the same reason and using linear extrapolation is $O(1)$. This must be done for each of the $n$ different quadrature nodes and weights, which shows that the total amount of operations is of order $O(m n)$.

Remark 4.5. Although the total order of operations does not depend on how the initial values are obtained, the constant in the $O(m n)$ expression can be quite different. As shown in the Examples section, the method based on the asymptotic zero distribution is considerably faster than the one based on linear extrapolation when there are only a finite number of different poles. 


\section{Examples}

In this section we give several examples to illustrate the construction and use of rational Gauss-Chebyshev quadrature formulas. First we look at the cost of computing the nodes and weights, and compare the two methods based on Newton iteration and the method using the generalized eigenvalue problem (GEP) mentioned in Section 2, Then we look at how our quadrature formulas perform compared to some alternative formulas. All of the computations were done in Matlab 6.5 on a Pentium III (Coppermine) with a CPU speed of $733 \mathrm{MHz}$.

The following examples give timings for computing the nodes and weights up to machine precision, for several values of $n$ and different locations of the poles. For the two methods based on Newton iteration, we also give the maximum number of iterations required and the maximal distance between the initial values and the exact solutions, $\max _{k}\left|\theta_{k n}^{0}-\theta_{k n}\right|$. Note that for large $n$, these initial values are very close to the actual zeros. The spline approximation is computed with the Matlab function spline. The generalized eigenvalue problem is solved with polyeig, which seems to be of order $O\left(n^{3}\right)$. However, taking into account the special structure of the matrices involved, this can probably be reduced to $O\left(n^{2}\right)$ using a suitable implementation. Since the computations are almost identical for the three different weight functions in (3.1), we limit our attention to the first case. The notation in the tables is as follows: $T$ refers to the time (in seconds), $\Delta$ is the maximal distance between initial values and exact zeros, and $i$ is the maximum number of iterations. Subscripts refer to the method: $L E$ is the method based on linear extrapolation, $Z D$ the one based on asymptotic zero distribution, and $G E P$ is the generalized eigenvalue problem.

Example 5.1. The first example illustrates the simplest situation where all poles are equal to each other. In this case we can use the exact asymptotic zero distribution to obtain the initial values. First we take $\alpha_{k}=\alpha=10$. The results are shown in Table 1. Note how this table clearly shows that the complexity in the first two cases is $O(n)$, but the method based on the asymptotic zero distribution is more than ten times faster than the one based on linear extrapolation, even though the latter provides far more accurate initial values for the Newton iterations. The GEP was not solved for $n>1600$, because this was taking too long.

Next we take $\alpha=1.01$, so we have a multiple pole very close to the interval. Table 2 shows the results. Note that we need more iterations to converge to the exact values of the nodes, although this does not substantially influence the timings.

TABle 1 . Multiple pole in $\alpha=10$

\begin{tabular}{|c|c|c|c|c|c|c|c|}
\hline$n$ & $T_{L E}$ & $\Delta_{L E}$ & $i_{L E}$ & $T_{Z D}$ & $\Delta_{Z D}$ & $i_{Z D}$ & $T_{G E P}$ \\
\hline 100 & $4.26 e-02$ & $9.96 e-05$ & 3 & $5.40 e-03$ & $5.04 e-04$ & 3 & $3.69 e-01$ \\
200 & $7.88 e-02$ & $2.50 e-05$ & 3 & $3.16 e-03$ & $2.52 e-04$ & 3 & $2.84 e+00$ \\
400 & $1.33 e-01$ & $6.25 e-06$ & 3 & $4.63 e-03$ & $1.26 e-04$ & 3 & $2.70 e+01$ \\
800 & $2.71 e-01$ & $1.56 e-06$ & 3 & $7.74 e-03$ & $6.30 e-05$ & 3 & $3.51 e+02$ \\
1600 & $5.68 e-01$ & $3.91 e-07$ & 2 & $1.17 e-02$ & $3.15 e-05$ & 3 & $3.08 e+03$ \\
3200 & $1.55 e+00$ & $9.78 e-08$ & 2 & $2.44 e-02$ & $1.57 e-05$ & 2 & - \\
6400 & $4.03 e+00$ & $2.45 e-08$ & 2 & $5.32 e-02$ & $7.87 e-06$ & 2 & - \\
12800 & $1.56 e+01$ & $6.11 e-09$ & 2 & $9.71 e-02$ & $3.94 e-06$ & 2 & - \\
\hline
\end{tabular}


TABLe 2. Multiple pole in $\alpha=1.01$

\begin{tabular}{|c|c|c|c|c|c|c|c|}
\hline$n$ & $T_{L E}$ & $\Delta_{L E}$ & $i_{L E}$ & $T_{Z D}$ & $\Delta_{Z D}$ & $i_{Z D}$ & $T_{G E P}$ \\
\hline 100 & $4.81 e-02$ & $5.37 e-02$ & 4 & $3.63 e-03$ & $5.27 e-02$ & 4 & $5.77 e-01$ \\
200 & $1.04 e-01$ & $1.49 e-02$ & 4 & $3.60 e-03$ & $2.65 e-02$ & 4 & $2.46 e+00$ \\
400 & $1.68 e-01$ & $3.86 e-03$ & 3 & $5.05 e-03$ & $1.34 e-02$ & 4 & $2.40 e+01$ \\
800 & $3.07 e-01$ & $9.84 e-04$ & 3 & $8.61 e-03$ & $6.70 e-03$ & 4 & $3.10 e+02$ \\
1600 & $6.05 e-01$ & $2.48 e-04$ & 3 & $1.20 e-02$ & $3.35 e-03$ & 3 & $3.05 e+03$ \\
3200 & $1.55 e+00$ & $6.24 e-05$ & 3 & $2.68 e-02$ & $1.68 e-03$ & 3 & - \\
6400 & $4.02 e+00$ & $1.56 e-05$ & 2 & $5.70 e-02$ & $8.39 e-04$ & 3 & - \\
12800 & $1.54 e+01$ & $3.91 e-06$ & 2 & $1.20 e-01$ & $4.20 e-04$ & 3 & - \\
\hline
\end{tabular}

TABLE 3. Two different poles $\alpha_{2 k}=1.1$ and $\alpha_{2 k+1}=-5$

\begin{tabular}{|c|c|c|c|c|c|c|c|}
\hline$n$ & $T_{L E}$ & $\Delta_{L E}$ & $i_{L E}$ & $T_{Z D}$ & $\Delta_{Z D}$ & $i_{Z D}$ & $T_{G E P}$ \\
\hline 100 & $4.58 e-02$ & $9.41 e-04$ & 3 & $1.28 e-02$ & $8.65 e-03$ & 4 & $3.48 e-01$ \\
200 & $9.29 e-02$ & $2.36 e-04$ & 3 & $1.26 e-02$ & $4.33 e-03$ & 4 & $2.53 e+00$ \\
400 & $1.71 e-01$ & $5.92 e-05$ & 3 & $1.57 e-02$ & $2.17 e-03$ & 4 & $2.60 e+01$ \\
800 & $2.85 e-01$ & $1.48 e-05$ & 3 & $2.06 e-02$ & $1.08 e-03$ & 3 & $3.25 e+02$ \\
1600 & $6.05 e-01$ & $3.71 e-06$ & 3 & $3.47 e-02$ & $5.42 e-04$ & 3 & $3.01 e+03$ \\
3200 & $1.55 e+00$ & $9.27 e-07$ & 3 & $6.40 e-02$ & $2.71 e-04$ & 3 & - \\
6400 & $4.20 e+00$ & $2.32 e-07$ & 2 & $1.12 e-01$ & $1.35 e-04$ & 3 & - \\
12800 & $1.60 e+01$ & $5.80 e-08$ & 2 & $2.31 e-01$ & $6.77 e-05$ & 3 & - \\
\hline
\end{tabular}

Example 5.2. Before we move to the most general case of arbitrary poles, we look at the situation where there are only two different poles (which is essentially the same as the case of any finite number of different poles). We can no longer use the exact zero distribution, but must instead construct the spline approximation. For the number of interpolation points we take $m=50$ (increasing this number to $m=500$ did not make any difference at all). The poles are $\alpha_{2 k}=1.1$ and $\alpha_{2 k+1}=-5$. The results are shown in Table 3 .

Example 5.3. Table 4 gives some timings for computing the nodes and weights in the case of arbitrary poles $\alpha_{k}=1 /\left(2 \tilde{\alpha}_{k}-1\right)$, where the points $\tilde{\alpha}_{k}$ are randomly chosen from a uniform distribution on $[0,1]$. In fact, with this knowledge we could use the exact asymptotic zero distribution to obtain the initial values, but we do not do this, and instead use the procedure based on the spline approximation. Note that the algorithm in this case is of order $O\left(n^{2}\right)$. It is also interesting to note that the method based on the asymptotic zero distribution is now slower than the one based on linear extrapolation. This is probably due to the fact that we can no longer use Matlab's sum command to compute the functions $f_{n}$ and $f_{n}^{\prime}$, but instead use a loop to compute this sum (otherwise the storage requirements would become too large; we would have to store an $n \times n$-matrix). Again this shows how much these timings depend on the specific implementation.

In the remaining examples we shall be concerned with the accuracy of these formulas and compare them to some alternative quadrature rules. We estimate 
TABLE 4. Random poles

\begin{tabular}{|c|c|c|c|c|c|c|c|}
\hline$n$ & $T_{L E}$ & $\Delta_{L E}$ & $i_{L E}$ & $T_{Z D}$ & $\Delta_{Z D}$ & $i_{Z D}$ & $T_{G E P}$ \\
\hline 100 & $1.49 e-01$ & $6.28 e-04$ & 3 & $1.49 e-01$ & $4.20 e-03$ & 3 & $3.72 e-01$ \\
200 & $2.85 e-01$ & $1.18 e-04$ & 3 & $2.83 e-01$ & $3.35 e-04$ & 3 & $3.07 e+00$ \\
400 & $6.80 e-01$ & $3.63 e-05$ & 3 & $9.45 e-01$ & $5.52 e-04$ & 3 & $2.52 e+01$ \\
800 & $1.51 e+00$ & $8.36 e-06$ & 3 & $3.48 e+00$ & $5.01 e-04$ & 3 & $3.50 e+02$ \\
1600 & $5.10 e+00$ & $1.98 e-06$ & 3 & $1.34 e+01$ & $4.34 e-04$ & 3 & $3.22 e+03$ \\
3200 & $1.96 e+01$ & $5.78 e-07$ & 3 & $5.71 e+01$ & $3.52 e-04$ & 3 & - \\
6400 & $8.64 e+01$ & $1.37 e-07$ & 3 & $2.25 e+02$ & $1.27 e-04$ & 3 & - \\
12800 & $4.03 e+02$ & $3.21 e-08$ & 3 & $9.76 e+02$ & $9.45 e-05$ & 3 & - \\
\hline
\end{tabular}

TABLE 5. Relative error for $I\left(f_{1}\right)$ with $\omega=1.1$

\begin{tabular}{|c|c|c|}
\hline$n$ & ORF & classical \\
\hline 2 & $1.42 e-02$ & $3.81 e-01$ \\
4 & $7.68 e-05$ & $7.41 e-02$ \\
8 & $1.24 e-12$ & $2.19 e-03$ \\
16 & $2.45 e-16$ & $1.82 e-06$ \\
32 & $3.68 e-16$ & $1.24 e-12$ \\
\hline
\end{tabular}

integrals of the form

$$
I\left(f_{i}\right)=\int_{-1}^{1} \frac{f_{i}(x)}{\sqrt{1-x^{2}}} d x
$$

for different functions $f_{i}$.

Example 5.4. The first function $f_{1}(x)$ to be considered is taken from [11, 12]. However, Gautschi computes the integral $\int_{-1}^{1} f_{1}(x) d x$, so we cannot compare our results to his. In this case we have

$$
f_{1}(x)=\frac{\pi x / \omega}{\sin (\pi x / \omega)}
$$

which has simple poles at the integer multiples of $\omega$. First we take $\omega=1.1$ and set $A=\{\omega,-\omega, 2 \omega,-2 \omega, \ldots\}$. Table 5 gives the relative error for several values of $n$. The left column shows the results for our quadrature formula, and the right column corresponds to the classical Chebyshev rule

$$
\int_{-1}^{1} \frac{f(x)}{\sqrt{1-x^{2}}} d x \approx \frac{\pi}{n} \sum_{k=1}^{n} f\left(x_{n k}\right)
$$

where $x_{n k}$ are the zeros of $T_{n}(x)$. Next we take $\omega=1.001$, so we have a pole very close to the interval of integration. The results are shown in Table 6, which clearly shows the advantage of using rational quadrature formulas.

Example 5.5. This example is taken from [13, where the author constructs (among other things) a quadrature formula which integrates exactly functions of the form

$$
f(x)=\frac{p_{2 r-1}(x)}{(x+\lambda)^{r}}, \quad p_{2 r-1} \in \mathcal{P}_{2 r-1}
$$


TABLE 6. Relative error for $I\left(f_{1}\right)$ with $\omega=1.001$

\begin{tabular}{|c|c|c|}
\hline$n$ & ORF & classical \\
\hline 2 & $4.99 e-03$ & $9.36 e-01$ \\
4 & $5.44 e-05$ & $8.46 e-01$ \\
8 & $1.90 e-12$ & $6.75 e-01$ \\
16 & $1.19 e-14$ & $3.97 e-01$ \\
32 & $1.37 e-14$ & $1.11 e-01$ \\
\hline
\end{tabular}

TABLE 7. Relative error for $I\left(f_{2}\right)$ with $\lambda=1.01$ and $r=2$

\begin{tabular}{|c|c|c|c|}
\hline$n$ & ORF & Sri Ranga & classical \\
\hline 2 & $5.41 e-04$ & $8.83 e-05$ & $9.77 e-01$ \\
4 & $6.11 e-07$ & $6.31 e-07$ & $8.93 e-01$ \\
8 & $1.29 e-15$ & $4.97 e-12$ & $5.64 e-01$ \\
16 & $6.46 e-16$ & $2.37 e-15$ & $1.15 e-01$ \\
\hline
\end{tabular}

with respect to the Chebyshev weight function. The nodes and weights can be computed from the classical Chebyshev formula. He then uses this formula to estimate $I\left(f_{2}\right)$ with

$$
f_{2}(x)=\frac{\exp (x)}{(x+\lambda)^{r}}
$$

for some values of $\lambda$ and $r$. We reproduce his results for $\lambda=1.01$ and $r=2$, and compare them to our method where we take $\alpha_{1}=\alpha_{2}=\ldots b=\alpha_{r}=-\lambda$ and $\alpha_{k}=\infty$ for $k>r$. The results are shown in Table 7 , where we also show the relative error for the classical Chebyshev rule. Convergence seems to be a little bit faster for our method.

Example 5.6. Now we look at the integral $I\left(f_{3}\right)$ with

$$
f_{3}(x)=\frac{1}{\sqrt{(x+3)(x+2)}} .
$$

This function has a branch cut on $[-3,-2]$ and, as noted by Van Assche in [16], is in fact a Stieltjes function given by

$$
f_{3}(x)=\frac{1}{\pi} \int_{-3}^{-2} \frac{1}{x-t} \frac{d t}{\sqrt{(3+t)(-2-t)}} .
$$

As is known, Stieltjes functions can be well approximated by rational functions with poles on the cut. We look at three different choices for the poles. In the first case we take $\alpha_{k}=-2.5$ for all $k$ (a multiple pole in the middle of the interval). Next we take two different poles, $\alpha_{2 k+1}=(1 / \sqrt{2}-5) / 2$ and $\alpha_{2 k}=(-1 / \sqrt{2}-5) / 2$ (these are the zeros of $T_{2}(x)$ transformed to the interval $\left.[-3,-2]\right)$. Finally we take all poles different from each other as in the second example of [16. They are the zeros of successive Chebyshev polynomials $T_{3^{m}}(x)$, transformed to $[-3,-2]$ and ordered in such a way that they are dense on this interval. For more information we refer to the article. The results are shown in Table 8 . Note that there is no need to take all poles different from each other and dense on the interval; the second case gives 
TABLE 8. Relative error for $I\left(f_{3}\right)$

\begin{tabular}{|c|c|c|c|c|}
\hline$n$ & ORF - case 1 & ORF - case 2 & ORF - case 3 & classical \\
\hline 2 & $2.71 e-05$ & $6.60 e-06$ & $2.34 e-05$ & $5.35 e-03$ \\
4 & $3.24 e-10$ & $1.57 e-11$ & $7.30 e-12$ & $1.90 e-05$ \\
6 & $3.94 e-15$ & $1.23 e-16$ & $2.46 e-16$ & $7.80 e-08$ \\
8 & $1.23 e-16$ & $3.70 e-16$ & $3.70 e-16$ & $3.44 e-10$ \\
\hline
\end{tabular}

TABLE 9. Relative error for $I\left(f_{4}\right)$ with $\alpha=1.001$

\begin{tabular}{|c|c|c|}
\hline$n$ & ORF & classical \\
\hline 100 & $2.12 e-03$ & $5.32 e-02$ \\
200 & $8.01 e-04$ & $5.75 e-02$ \\
400 & $2.01 e-14$ & $4.21 e-02$ \\
800 & $2.94 e-14$ & $1.13 e-02$ \\
\hline
\end{tabular}

perfectly satisfactory results as well, and the quadrature formula can be computed more efficiently, as mentioned before.

Example 5.7. In the previous examples we have only looked at functions which can be integrated with a small number of nodes. They serve to illustrate the theory, but the real strength of these quadrature formulas lies in the approximation of integrals which are very difficult to calculate with conventional methods and which require a very high number of nodes. As shown by Examples 5.15 .3 and Theorem 4.4, our quadrature formulas can be computed efficiently (especially for a finite number of different poles), even for a very high degree. We give one example where this is of much use. Take

$$
f_{4}(x)=\sin \left(\frac{1}{x^{2}-\alpha^{2}}\right)
$$

where $\alpha>1$ but very close to 1 . This function has an essential singularity in $x=\alpha$ and $x=-\alpha$ and is extremely oscillatory near these points. Since an essential singularity can be viewed as a pole of infinity multiplicity, this suggests taking $A=\{\alpha,-\alpha, \alpha,-\alpha, \ldots\}$ (a more intuitive reason is that the presence of poles near the boundary attracts the zeros to the endpoints, where the integrand is very oscillatory). For $\alpha=1.001$ the results are shown in Table 9. It is difficult to obtain an accuracy of more than approximately 14 digits because of heavy cancellation in the quadrature sum. To obtain this accuracy using the classical Chebyshev rule we needed $n=5000$ nodes; using our method $n=400$ suffices. For integrands whose evaluation is very difficult (for example the solution of a differential equation which has to be obtained numerically), this reduction in the number of function evaluations can be very important.

\section{Concluding Remarks}

We have presented a set of quadrature formulas which integrate rational functions with arbitrary poles with respect to a Chebyshev weight function. For a finite number of different poles, these formulas can be computed very efficiently in $O(n)$ 
operations. If there is only one multiple pole, the formulas from 13. provide similar results, but for the case where there are more poles, we do not know of any competitive formulas.

The last example suggests a method for the numerical integration of functions with essential endpoint singularities, using a suitable extrapolation scheme for $\alpha \rightarrow$ 1. This problem (in a more general form) is still under investigation.

To deduce the quadrature formulas in this paper, we have introduced a rational generalization of the Chebyshev polynomials. These are among the very few explicit examples of orthogonal rational functions on an interval (and the only ones for arbitrary poles). We note that the results for $\varphi_{n}^{(1)}(x)$ could also be deduced from the so-called Malmquist basis of rational functions orthogonal on the unit circle with respect to the Lebesgue measure. Some preliminary results for this case were obtained in [19].

\section{REFERENCES}

[1] A. Bultheel, C. Díaz Mendoza, P. González Vera, and R. Orive, On the convergence of certain Gauss-type quadrature formulas for unbounded intervals, Math. Comp. 69(230) (1999), 721747. MR.1651743 (2000i:65034)

[2] A. Bultheel, P. González Vera, E. Hendriksen, and O. Njåstad, Orthogonal rational functions, Cambridge Monographs on Applied and Computational Mathematics, vol. 5, Cambridge University Press, 1999. MR1676258 (2000c:33001)

[3] _ Orthogonal rational functions and quadrature on the real half line, J. Complexity 19(3) (2002), 212-230. MR.1984110 (2004d:41054)

[4] _ Orthogonal rational functions and tridiagonal matrices, J. Comput. Appl. Math. 153(1-2) (2003), 89-97. MR.1985681 (2004e:42040)

[5] C. Díaz Mendoza, P. González Vera, and M. Jiménez Paiz, Strong Stieltjes distributions and orthogonal Laurent polynomials with applications to quadratures and Padé approximation, Math. Comp. 74 (2005), 1843-1870.

[6] C. Díaz Mendoza, P. González Vera, M. Jiménez Paiz, and F. Cala Rodríguez, Orthogonal Laurent polynomials corresponding to certain strong Stieltjes distributions with applications to numerical quadratures, Math. Comp., posted on September 9, 2005, PII S 00255718(05)01781-3 (to appear in print).

[7] W. Gautschi, Construction of Gauss-Christoffel quadrature formulas, Math. Comp. 22 (1968), 251-270. MR0228171 (37:3755)

[8] - On the Construction of Gaussian Quadrature Rules from Modified Moments, Math. Comp. 24 (1970), 245-260. MR0285117 (44:2341a)

[9] _ On generating orthogonal polynomials, SIAM J. Sci. Stat. Comput. 3 (1982), 289317. MR0667829 (84e:65022)

[10] _ Orthogonal polynomials: applications and computation, Acta Numerica 5 (1996), 45-119. MR 1624591 (99j:65019)

[11] _ Algorithm 793: GQRAT - Gauss Quadrature for Rational Functions, ACM Trans. Math. Software 25 (1999), 113-139.

[12] _ Quadrature rules for rational functions, Numer. Math. 86(4) (2000), 617-633. MR1794345 (2002a:41030)

[13] A. Sri Ranga, Another quadrature rule of highest algebraic degree of precision, Numer. Math. 68 (1994), 283-294. MR 1283343 (95c:65047)

[14] P.N. Swarztrauber, On computing the points and weights for Gauss-Legendre quadrature, SIAM J. Sci. Comput. 24(3) (2002), 945-954. MR1950519 (2004a:65027)

[15] G. Szegő, Orthogonal polynomials, Am. Math. Soc. Colloq. Publ., vol. 23, Am. Math. Soc., Providence, RI, 1967. MR0310533 (46:9631)

[16] W. Van Assche and I. Vanherwegen, Quadrature formulas based on rational interpolation, Math. Comp. 61(204) (1993), 765-783. MR.1195424 (94a:65014)

[17] J. Van Deun and A. Bultheel, Orthogonal rational functions and quadrature on an interval, J. Comput. Appl. Math. 153(1-2) (2003), 487-495. MR.1985717(2004e:42043) 
[18] _ Ratio asymptotics for orthogonal rational functions on an interval, J. Approx. Theory 123 (2003), 162-172. MR.1990094 (2004j:41047)

[19] P. Van Gucht and A. Bultheel, A relation between orthogonal rational functions on the unit circle and the interval $[-1,1]$, Comm. Anal. Th. Continued Fractions 8 (2000), 170-182. MR.1789681 (2001h:42037)

Department of Computer Science, K.U.Leuven, B-3001 Heverlee, Belgium

E-mail address: joris.vandeun@cs.kuleuven.ac.be

Department of Computer Science, K.U.Leuven, B-3001 Heverlee, Belgium

E-mail address: adhemar.bultheel@cs.kuleuven.ac.be

Depto. Análisis Matemático, Univ. La Laguna, 38206 La laguna, Tenerife, Canary ISLANDS, SPAIN

E-mail address: pglez@ull.es 